\title{
La función de Gompertz-Makeham en la descripción y proyección de fenómenos demográficos*
}

\section{Héctor Ogaz Pierce**}

La función matemática de Gompertz-Makeham ha recibido una creciente atención por parte de algunos investigadores, tanto por su capacidad para describir fenómenos demográficos, como por su flexibilidad para proyectar sus tendencias. No obstante, para abordar diversas aplicaciones, se le ha empleado poco en la práctica, a diferencia de otro tipo de funciones.

El objetivo de este artículo es en primera instancia profundizar en el análisis de la aplicación de esta función, partiendo de su formulación y desarrollo matemático. En segundo término, probar un método iterativo para la obtención de parámetros, que permita obtener una función óptima que describa mejor el comportamiento de una población ante los fenómenos demográficos.

El estudio se llevó a cabo con el crecimiento de la población y las estructuras por edad tanto de la fecundidad como de la población económicamente activa. El resultado muestra lo versátil y útil que es trabajar con la función de Gompertz-Makeham, teniendo presente en todo momento el conocimiento de hipótesis y supuestos demográficos implicados.

Este artículo se centra en el análisis de la función matemática desarrollada por Gompertz y Makeham para la descripción, proyección y simulación de fenómenos demográficos. En la primera parte se mencionan algunos de los trabajos más conocidos sobre el tema; en la segunda se desarrolla la metodología matemática empleada; a continuación se presentan las aplicaciones y el análisis de los resultados obtenidos, enfatizando los aspectos demográficos inmersos en los fenómenos estudiados, y finalmente, en la última parte se exponen las conclusiones sobre el análisis de las bondades y deficiencias de la función de Gompertz-Makeham.

\section{Introducción}

En la investigación demográfica la descripción de los fenómenos demográficos mediante la formulación de métodos y modelos ma-

* Este trabajo es una síntesis de la tesis de maestria en demografía del autor, el cual agradece la dirección del profesor Alejandro Mina V., investigador de El Colegio de México.

** Unidad de Servicios Actuariales del IMSS. 
temáticos resulta indispensable para captar el patrón de comportamiento que revele las tendencias en su dinámica evolutiva, a fin de estar en posibilidad de predecir lo que se esperaría en el futuro, de continuar con las condiciones que provocan la ocurrencia del suceso demográfico en estudio.

A Gompertz y Makeham correspondió realizar uno de los principales esfuerzos para desarrollar alguna función matemática que describiera en primera instancia el fenómeno de la sobrevivencia. Actualmente algunos investigadores, como el profesor Albino Bocaz, han demostrado que, entre otras aplicaciones, la función matemática desarrollada por Gompertz y Makeham describe apropiadamente las tasas de fecundidad acumuladas por edad y resume proyecciones de población por grupos de edad en relación con diversas hipótesis hechas acerca de la evolución, en el tiempo, de la mortalidad y la fecundidad.

A su vez, García y Mina comprueban que la función de Gompertz-Makeham describe adecuadamente las estructuras acumuladas por edad y sexo de la población económicamente activa [PEA] y que también permite desagregar, de grupos quinquenales de edad a edades individuales, una vez efectuado el ajuste descriptivo. Asimismo Mina ha comprobado que la ley de Makeham describe adecuadamente el comportamiento del fenómeno de la nupcialidad, específicamente el riesgo de casarse.

A pesar del interés de varios investigadores en México, por este tipo de funciones matemáticas, hasta la fecha se ha trabajado poco con la función de Gompertz-Makeham, soslayando su riqueza para representar un fenómeno demográfico. Debido a ello, en este trabajo se pretende profundizar en el análisis de la aplicación de dicha función matemática, enfatizando lo relevantes que son los elementos y supuestos demográficos implicados, sin caer en la aplicación mecánica del modelo empleado.

Al realizar la aplicación de los ajustes descriptivos se utilizó por primera vez en este tipo de estudios un método iterativo, que permite en algunos casos y situaciones, lograr una función óptima; ésta registra el comportamiento de una población ante los fenómenos demográficos y por tanto posibilita proyectar su tendencia con menor sesgo.

En suma, este estudio se efectuó pensando en diferentes situaciones, que implican las siguientes aplicaciones: el crecimiento de la población y las estructuras por edad de la fecundidad y de la población económicamente activa ocupada.

Un aspecto esencial que se debe considerar en la descripción de un fenómeno demográfíco, es el disponer de información confiable, para lo cual se requiere de evaluar y en su caso ajustar y 
corregir los datos con los cuales se vaya a trabajar; sin embargo, para el caso que nos ocupa no se realizó tarea alguna al respecto, dado que el objeto de estudio es el análisis de la función de Gompertz-Makeham y por tanto la información utilizada se extrajo directamente de las publicaciones ofïciales, sin realizar evaluaciones previas.

\section{Metodología}

A través del enfoque de funciones de sobrevivencia, en esta parte se presenta la función de Gompertz-Makeham, un método para calcular sus parámetros y un algoritmo de optimización de la misma.

\section{Funciones de sobrevivencia}

La sobrevivencia depende principalmente de aspectos biológicos, en consecuencia, el elemento primordial para su medición es la edad; es decir, los procesos biológicos del individuo se realizan en el tiempo f́́sico o absoluto, pero se manifiestan en relación con la edad, como medida del tiempo interno del individuo.

Lo importante del tiempo es la percepción que se tiene de las cosas a través de su duración propia. Duración que se forma mediante el surgimiento de un momento de tiempo a otro, merced a la finitud de la velocidad de cambio de cualquier proceso.

La medida del fenómeno de la sobrevivencia va ligada a la idea del tiempo con un amplio significado matemático, donde las principales relaciones matemáticas se expresan a través de dos variables aleatorias, mismas que representan los hechos de sobrevivir a una edad determinada o de no superarla.

Así que la probabilidad de no sobrevivir a la edad $x$ vendrá dada por la función de distribución acumulativa

$$
F[x]=P(\varepsilon \leqq x)
$$

mientras que por ser el suceso contrario, la probabilidad de superar esta edad es

$$
S(x)=P[\varepsilon>x]=1-F(x)
$$

donde $\varepsilon$ se define como la edad a la muerte.

Si se conoce la función de distribución acumulativa $F(x)$ o la 
complementaria $S(x)$ identificada como función de sobrevivencia, puede determinarse una función de mortalidad $f(x)$, al derivar $F(x)$ o $S(x)$, sólo que, en el último caso, con signo cambiado, tal y como se observa a continuación:

$$
f(x)=\frac{d F[x]}{d x}=-\frac{d S(x)}{d x}
$$

En demografía, la llamada fuerza de mortalidad o tasa instantánea de mortalidad $\mu_{x}$ es evidentemente la densidad de la distribución condicionada al suceso de haber superado la edad $\mathrm{x}$ :

$$
\mu_{x}=\frac{f(x)}{S(x)}=-\frac{d S(x)}{S(x) d x}=-\frac{d \ln S(x)}{d x}
$$

La relación [4] es importante, porque expresa cómo a la fuerza de la mortalidad se opone una fuerza de vitalidad, como equilibrio vital relativo a cada edad. Por tanto la modelización de funciones de sobrevivencia se hace fundamentalmente sobre $\mu_{\mathrm{x}}$.

\section{Función de Gompertz-Makeham}

\section{Gompertz}

Este autor se planteó como objetivo observar cómo se extinguía una población, así que después de encontrar que conforme pasa el tiempo la resistencia del hombre a la muerte decrece proporcionalmente a la misma, Gompertz definió la resistencia del hombre a la muerte como el recíproco de la tasa instantánea de mortalidad $\left[1 / \mu_{\mathrm{x}}\right]$ :

$$
\frac{d\left[1 / \mu_{x}\right]}{d x}=-h \frac{1}{\mu_{x}}
$$

siendo $h$ la tasa a la cual decrece la resistencia del hombre a la muerte.

A partir de la hipótesis planteada por Gompertz en [5] se obtiene una función de sobrevivencia, de la siguiente manera:

considerada constante la relación $1 / \mu_{\mathrm{x}}$ e integrando [5]:

$$
\frac{\int \frac{d\left[1 / \mu_{x}\right]}{d x} \cdot d x}{\left[1 / \mu_{x}\right]}=-h \int d x
$$


y obtenida la integración se tiene

$$
\ln \frac{B}{\mu_{\mathrm{x}}}=-\mathrm{hx}
$$

con $B=e^{\left(C_{1}-C_{2}\right)}$ (con $C_{1}$ y $C_{2}$ como constantes de integración). Para de ahí despejar $\mu_{\mathrm{x}}$ :

$$
\mu_{\mathrm{x}}=B C^{\mathrm{x}}
$$

donde $C=e^{h}$.

El resultado obtenido por Gompertz describió la fuerza de mortalidad por primera vez como una función exponencial y que exclusivamente considera las causas de muerte dependientes de la edad.

Ahora bien, como ya es sabido, la tasa instantánea de mortalidad $\mu_{\mathrm{x}}$ mide el cambio relativo en ese fenómeno y por tanto se expresa por

$$
\mu_{\mathrm{x}}=\lim _{\delta \rightarrow 0} \frac{l(\mathrm{x}+\delta)-l(\mathrm{x})}{\delta l[\mathrm{x})}=-\frac{\mathrm{d}}{\mathrm{dx}} \ln l(\mathrm{x})
$$

donde $][x]$ es una función continua de sobrevivencia relativa a una tabla de mortalidad y se refiere a los sobrevivientes de un conjunto inicial de recién nacidos.

Al combinar las expresiones (8) y (9), se tiene

$$
-\frac{d}{d x} \ln l[x)=B C^{x}
$$

ecuación diferencial que relaciona la función de sobrevivencia l[x] con los parámetros hallados por Gompertz para definir la fuerza de la mortalidad.

Integrando la ecuación (10)

$$
\int \frac{d}{d x} \ln l(x) d x=-\int B C^{x} d x
$$

se obtiene

$$
\ln l(x)=C^{x} \ln g+\ln k
$$


donde $k=e^{\left(C 1-C^{-2)}\right.}$ y $\ln g=-B / \ln C$, siendo $C_{1}$ y $C_{2}$ las constantes de integración. Para de ahí llegar a la expresión conocida como la ley de Gompertz:

$$
l(x)=K g^{\cdot x}
$$

\section{Makeham}

A su vez, Makeham retoma lo desarrollado por Gompertz e incorpora a la función de sobrevivencia, las causas de muerte independientes de la edad. Por lo que de (8), Makeham plantea para la fuerza de mortalidad, la expresión:

$$
\mu_{\mathrm{x}}=A+B C^{\mathrm{x}}
$$

donde $A$ se asocia al efecto de las causas de muerte fortuitas. Al sustituir el valor de $\mu_{\mathrm{x}}$ de (14) en [9], se tiene

$$
-\frac{d}{d x} \ln l[x)=A+B C^{x}
$$

Nuevamente se tiene una ecuación diferencial que relaciona la función de sobrevivencia $\mathrm{l}$ [ $\mathrm{x}$ ) con los parámetros propuestos por Makeham para definir la fuerza de la mortalidad. Procediendo a la integración de (15)

$$
\int \frac{d}{d x} \ln l(x) d x=-\int\left(A+B C^{x}\right) d x
$$

se obtiene

$$
\ln l(x)=x \ln s+C^{x} \ln g+\ln k
$$

$\operatorname{con} k=e^{\left(C_{1}-C_{2}\right)}, \ln g=-B / \ln C$ y $\ln s=-A$, siendo $C_{1}$ y $C_{2}$ las constantes de integración.

Finalmente se llega a la expresión conocida como la ley de Gompertz-Makeham:

$$
l[x)=K s^{x} g^{x}
$$

Con lo cual, las dos funciones de sobrevivencia, para el caso general son: 


$$
\begin{array}{ll}
\text { Gompertz: } \quad Y(x)=K b^{d x} \\
\text { Gompertz-Makeham: } Y(x)=K a^{x} b^{d^{x}}
\end{array}
$$

\section{Método de los grupos no superpuestos}

Ahora, para el cálculo de los parámetros $(K, a, b, d)$ de la función de Gompertz-Makeham, se procede de la siguiente forma:

i) Dados $n$ valores observados, cada uno de ellos dependientes de la variable $x$ relativa a la edad:

$$
\left\{Y^{0}[i)\right\}_{i=0,1,2, \ldots n}
$$

se tiene el supuesto fundamental:

$$
\begin{gathered}
\text { para toda } i \ldots Y_{i} K a^{i} b^{d^{i}} \\
\text { se sigue que } \ln Y_{i}^{0}=\ln k+i \ln a+d^{i} \ln b
\end{gathered}
$$

ii) Se dividen los datos observados en 4 grupos de igual tamaño $(m)$, cuidando que los grupos no sean superpuéstos, es decir que la separación en observaciones sucesivas no se traslape. Por tanto:

Primer grupo: $\left\{Y^{0}[i]\right\}_{j=0,1,2, \ldots,(m-1)}$

Segundo grupo: $\left\{Y^{0}(i)\right\}_{i=m, m+1, m+2, \ldots,(2 m-1)}$

Tercer grupo: $\left\{Y^{0}[i]\right\}_{i=2 m, 2 m+1,2 m+2, \ldots,(3 m-1)}$

Cuarto grupo: $\left\{Y^{0}(i)\right\}_{i=3 m, 3 m+1,3 m+2, \ldots,(4 m-1)}$

iii) Se obtienen los logaritmos de $\left\{Y^{0}[i]\right\}$ y las 4 sumas correspondientes a cada grupo de sus in $\left\{Y^{0}(i)\right\}$ :

$$
\begin{aligned}
& S_{0}=\sum_{i=0}^{m-1} \ln Y^{0}(i)=m \ln K+\ln a \sum_{i=0}^{m-1} i+\ln b \sum_{j=0}^{m-1} d^{i} \\
& S_{1}=\sum_{j=m}^{2 m-1} \ln Y^{0}(i)=m \ln K+\ln a \sum_{i=m}^{2 m-1} i+\ln b \sum_{i=m}^{2 m-1} d^{i} \\
& S_{2}=\sum_{i=2 m}^{3 m-1} \ln Y^{0}(i)=m \ln K+\ln a \sum_{i=2 m}^{3 m-1} i+\ln b \sum_{j=2 m}^{3 m-1} d^{i} \\
& S_{3}=\sum_{i=3 m}^{4 m-1} \ln Y^{0}(i)=m \ln K+\ln a_{i=3 m}^{4 m-1} i+\ln b \sum_{i=3 m}^{4 m-1} d^{i}
\end{aligned}
$$


Dadas las relaciones anteriores, se observa que la $\Sigma i$ corresponde a una serie aritmética, mientras que la $\Sigma d^{l}$ a una serie geométrica, por lo que

$S_{0}=m \ln K+\frac{m(m-1)}{2} \ln a+\frac{1-d^{m}}{1-d} \ln b$

$S_{1}=m \ln K+\left[m^{2}+\frac{m(m-1)}{2}\right] \ln a+d^{m}\left(\frac{1-d^{m}}{1-d^{-}}\right) \ln b$

$S_{2}=m \ln K+\left[2 m^{2}+\frac{m(m-1)}{2}\right] \ln a+d^{2 m}\left(\frac{1-d^{m}}{1-d}\right) \ln b$

$S_{3}=m \ln K+\left[3 m^{2}+\frac{m(m-1)}{2}\right] \ln a+d^{3 m}\left(\frac{1-d^{m}}{1-d}\right) \ln b$

iv) Se obtienen las primeras y segundas diferencias basándose en las últimas cuatro relaciones:

diferencias

primeras

i $S_{i}$

$\Phi S_{i}$

$0 S_{0}$

$1 S_{1}$

$$
m^{2} \ln a+\frac{\left(d^{m}-1\right)^{2}}{d-1} \ln b
$$

$m^{2} \ln a+d^{m} \frac{\left(d^{m}-1\right)^{2}}{d-1} \ln b$

$2 \mathrm{~S}_{2}$

$$
m^{2} \ln a+d^{2 m} \frac{\left(d^{m}-1\right)^{2}}{d-1} \ln b
$$

$3 \quad S_{3}$

v) una vez conocidas dichas diferencias, se calculan los parámetros de la siguiente forma:

$$
\begin{gathered}
d=\left(\frac{\Phi^{2} S_{1}}{\Phi^{2} S_{0}}\right)^{1 / m} \\
b=\exp \left\{\frac{\Phi^{2} S_{0}(d-1)}{\left(d^{m}-1\right)^{3}}\right\} \\
a=\exp \left\{\frac{1}{m^{2}}\left[\Phi S_{0}-\frac{\left(d^{m}-1\right)^{2}}{d-1} \ln b\right]\right\}
\end{gathered}
$$


y para determinar $K$ se impone la condición de mínimos cuadrados de las diferencias entre los valores observados y los teóricos

$$
(D)^{2}=\sum_{i=0}^{n}\left(Y_{(i)}^{0}-K V_{(i)}\right)^{2}
$$

donde $V_{(i)}=a^{i} b^{d i}$ y $D$ representa las diferencias.

Para obtener un mínimo, se deriva con respecto a $\mathrm{K}$ y se iguala a 0 :

$$
\frac{\partial D^{2}}{\partial K}=2 \sum_{i=0}^{n}\left(Y_{(i)}^{0}-K V_{(i)}\right)\left(-V_{(i)}\right)=0
$$

y se despeja $K$

$$
K=\frac{\sum_{i=0}^{n} Y_{(i)}^{0} V_{(i)}}{\sum_{j=0}^{n} V_{(i)}^{2}}
$$

Método iterativo para optimizar el ajusto de la función de Gompertz-Makeham

Dada la función

$$
Y_{(i)}=K a^{i} b^{d i}
$$

y conocidos los parámetros $K, a, b, d$ se busca obtener una nueva función de Gompertz-Makeham, con los nuevos parámetros

$$
Y_{(i)}^{N}=K^{N} a^{N i} b^{N} d^{N i}
$$

i) De la relación (22), se producen pequeños cambios en los valores de los parámetros para generar una pequeña variación dy

$$
\frac{d y}{y}=\frac{d K}{K}+i \frac{d a}{a}+d^{i} \frac{d b}{b}+i d^{i} \ln b \frac{d d}{d}
$$

Si se define por un lado:

$$
C_{2}=\frac{d K}{K} ; C_{4}=\frac{d b}{b}
$$




$$
C_{3}=\frac{d a}{a} ; C_{5}=\frac{d d}{d} \ln b
$$

y por otro:

$$
\begin{gathered}
x_{1}=d y ; x_{2}=y ; x_{3}=i\left(x_{2}\right) \\
x_{4}=x_{2} d^{i} ; x_{5}=x_{3} d^{i}
\end{gathered}
$$

entonces la relación (38) adquiere la forma linealizada

$$
x_{1}=C_{2} x_{2}+C_{3} x_{3}+C_{4} x_{4}+C_{5} x_{5}
$$

es decir, la diferencia entre los valores observados y los teóricos se refleja en $d y$, por lo que $C_{2}, C_{3}, C_{4}$ y $C_{5}$ se determinan como coeficientes de regresión lineal con el método de mínimos cuadrados. ${ }^{1}$

ii) Las ecuaciones normales que en forma matricial quedan:

$$
\left[\begin{array}{l}
\sum x_{1} x_{2} \\
\sum x_{1} x_{3} \\
\sum x_{1} x_{4} \\
\sum x_{1} x_{5}
\end{array}\right]=\left[\begin{array}{l}
\sum x_{2} x_{2}+\sum x_{2} x_{3}+\sum x_{2} x_{4}+\sum x_{2} x_{5} \\
\sum x_{2} x_{3}+\sum x_{3} x_{3}+\sum x_{3} x_{4}+\sum x_{3} x_{5} \\
\sum x_{2} x_{4}+\sum x_{3} x_{4}+\sum x_{4} x_{4}+\sum x_{4} x_{5} \\
\sum x_{2} x_{5}+\sum x_{3} x_{5}+\sum x_{4} x_{5}+\sum x_{5} x_{5}
\end{array}\right]\left[\begin{array}{l}
C_{2} \\
C_{3} \\
C_{4} \\
C_{5}
\end{array}\right]
$$

y en forma matricial condensada

$$
b=A c
$$

donde $b$ es un vector columna de $4 \times 1$, con los productos de $x_{1}$ con $x_{2}, x_{3}, x_{4}$ y $x_{5}$.

A es una matriz de $4 \times 4$ con los pares de productos de las variables $\mathrm{x}_{2}, \mathrm{x}_{3}, \mathrm{x}_{4}$ y $\mathrm{x}_{3}$. y $C_{3}$.

c es un vector columna de $4 \times 1$, con los valores de $C_{2}, C_{3}, C_{4}$

Que al emplear álgebra matricial se obtienen los valores del vector $\mathbf{c}$

$$
c=A^{-1} b
$$

${ }^{1}$ Cabe notar que al linealizar un modelo no lineal, pueden no cumplirse los supuestos básicos de la regresión. Por ejemplo, al violarse el supuesto esencial de la regresión lineal dado por la esperanza matemática del error estadístico o perturbación estocástica igual a cero, con la varianza de dicho término igual a un número positivo y constante $\left(\sigma^{2}\right)$, los estimadores de mínimos cuadrados dejan de ser eficientes. 
donde $A^{-1}$ es la matriz inversa de $A$, siempre que exista.

iii) Enseguida se calculan los nuevos valores de los parámetros

$$
\begin{aligned}
K^{N} & =K\left(1+C_{2}\right] \\
a^{N} & =a\left(1+C_{3}\right] \\
b^{N} & =b\left[1+C_{4}\right] \\
d^{N}=d & \left(1+\left(C_{5} / l n b\right]\right)
\end{aligned}
$$

iv) Esto permite calcular nuevos valores teóricos y nuevas diferencias entre éstos y los valores observados que se asimilan a dy. Con esto se calculan nuevas correlaciones a $K, a, b, d$ y continúa el proceso iterativo hasta lograr una determinada convergencia en dichos valores.

En general, si $K_{i}, a_{i}, b_{i}$ y $d_{i}$ son valores de la iteración $i$, los valores de esos parámetros a la iteración $i+1$ serán

$$
\begin{gathered}
K_{i+1}=\left(1+C_{2 i}\right) K_{i} \\
a_{i+1}=\left(1+C_{3 i}\right) a_{i} \\
b_{i+1}=\left(1+C_{4 i}\right) b_{i} \\
d_{i+1}=\left(1+\left(C_{5 i} / \ln b_{i}\right)\right) d_{i}
\end{gathered}
$$

\section{Aplicaciones y resultados}

Con los aspectos teóricos concernientes a la función matemática de Gompertz-Makeham, se procede a la aplicación de ésta en la descripción y proyección de fenómenos demográficos como son: el crecimiento de la población y las estructuras por edad de la fecundidad y de la población económicamente activa ocupada.

En cada aplicación se realiza una breve exposición de los aspectos demográficos implicados, como elementos que justifiquen la necesidad de efectuar la descripción y proyección del fenómeno demográfico en estudio.

A su vez, en cada caso se calculó la función de Gompertz-Makeham a través de la estimación de sus parámetros; luego se buscó una función óptima, se efectuó la desagregación de la función acumulada y la proyección, ya fuera directamente con la función determinada o bien mediante la simulación de algunas alternativas variando los parámetros de la función de Gompertz-Makeham.

De tal forma, sin perder de vista los aspectos mencionados de la dinámica demográfica, se llevó a cabo el análisis de los resultados obtenidos, con el propósito de fundamentar el planteamiento de que la función de Gompertz-Makeham puede describir el com- 
portamiento de diversas poblaciones ante diferentes fenómenos demográficos y, asimismo, ser útil para proyectar tal dinámica demográfica.

\section{Descripción del crecimiento de la población en México}

En cuanto a volumen, la evolución demográfica de la población mexicana durante el presente siglo refleja un proceso de intenso crecimiento. El incremento alcanzado se debe a una fuerte disminución en la mortalidad y a la permanencia de una alta fecundidad, aunque el ritmo de este crecimiento ha disminuido a partir de los años setenta por la declinación que se ha dado en la fecundidad.

Para tener una idea de la velocidad de crecimiento, en el cuadro 1 se presentan las tasas de crecimiento intercensal de la población, como cifras que representan un promedio de lo ocurrido en el decenio y por tanto se refieren aproximadamente a la mitad del periodo. Para la estimación de las tasas se consideró la ley exponencial simple, caso particular de la ley de GompertzMakeham cuando el parámetro b se iguala a la unidad.

Éste es un proceso impresionante porque cuando México crecía por arriba de $3 \%$ (décadas cincuenta y sesenta), su población se duplicaba en 22 años aproximadamente; mientras que ahora que crece ligeramente abajo de $2 \%$, el proceso de duplicación se realizará más allá de los 35 años. Esto último muestra un cambio excepcional en la dinámica del crecimiento de la población. Sin embargo, el incremento en números absolutos continúa siendo significativo, al grado de que representa un enorme desafío, en espera de respuestas y soluciones acerca del desarrollo social, económico, político y cultural del país.

De ahí que la descripción adecuada del crecimiento de la población, sea importante para disponer de una base que permita proyectar tendencias y situaciones futuras; así como para sopesar las consecuencias de las decisiones presentes, que enfrenten el proceso de desenvolvimiento demográfico.

Con el fin de describir el crecimiento poblacional acumulado con la función de Gompertz-Makeham se tomaron como referencia los montos totales de población arrojados por los Censos Generales de Población y Vivienda de 1921 a 1990 [de este último sólo los resultados preliminares). Las cifras, mediante la ley de crecimiento exponencial simple, se "recorrieron" al 30 de junio de cada año censal. Asimismo, como los años de levantamiento censal coinciden con el inicio de década, a excepción de 1921, se pro- 
CUADRO 1

\begin{tabular}{ccc}
\hline Año censal & Población & $\begin{array}{c}\text { Tasa de crecimiento [\%] } \\
\text { (promedio de la década) }\end{array}$ \\
\hline 1921 & 14334780 & 1.60 \\
1930 & 16552722 & 1.72 \\
1940 & 19653552 & 2.72 \\
1950 & 25791017 & 3.03 \\
1960 & 34923129 & 3.23 \\
1970 & 48225238 & 3.27 \\
1980 & 66846833 & 1.94 \\
1990 & 81140922 & \\
\hline
\end{tabular}

Fuente: Censos Generales de Población y Vivienda. 1921-1990.

\section{CUADRO 2}

México 1920-1990: descripción del crecimiento poblacional acumulado mediante la función de Gompertz-Makeham

\begin{tabular}{llcr}
\hline & \multicolumn{2}{c}{ Población acumulada } & $\begin{array}{c}\text { Variación } \\
\text { Año }\end{array}$ \\
\cline { 2 - 3 } & observada & estimada & 2.71 \\
\hline 1920 & 13993424 & 14382928 & -3.39 \\
1930 & 30583502 & 29579302 & -0.90 \\
1940 & 50404767 & 49952792 & 0.31 \\
1950 & 76249358 & 76484263 & 0.26 \\
1960 & 111246230 & 111536360 & -0.86 \\
1970 & 160117797 & 158756859 & -1.70 \\
1980 & 227062782 & 223260566 & 1.09 \\
1990 & 308694622 & 312095173 & \\
\hline
\end{tabular}

CUADRO 3

México 1920-1990: desagregación del crecimiento poblacional acumulado mediante la función de Gompertz-Makeham

\begin{tabular}{lllr}
\hline & \multicolumn{2}{c}{ Población al 30 de junio } & \\
\cline { 2 - 3 } Año & observada & estimada & $\begin{array}{c}\text { Variación } \\
\text { (porcentaje) }\end{array}$ \\
\hline 1920 & 13993424 & 14382928 & 2.71 \\
1930 & 16590078 & 15196374 & -9.17 \\
1940 & 19821266 & 20373490 & 2.71 \\
1950 & 25844590 & 26531471 & 2.59 \\
1960 & 34996872 & 35052097 & 0.16 \\
1970 & 48871567 & 47220500 & -3.50 \\
1980 & 66944985 & 64503707 & -3.78 \\
1990 & 81631840 & 88834606 & 8.11 \\
\hline
\end{tabular}




\section{GRÁFICA 1}

México 1920-1990: crecimiento población

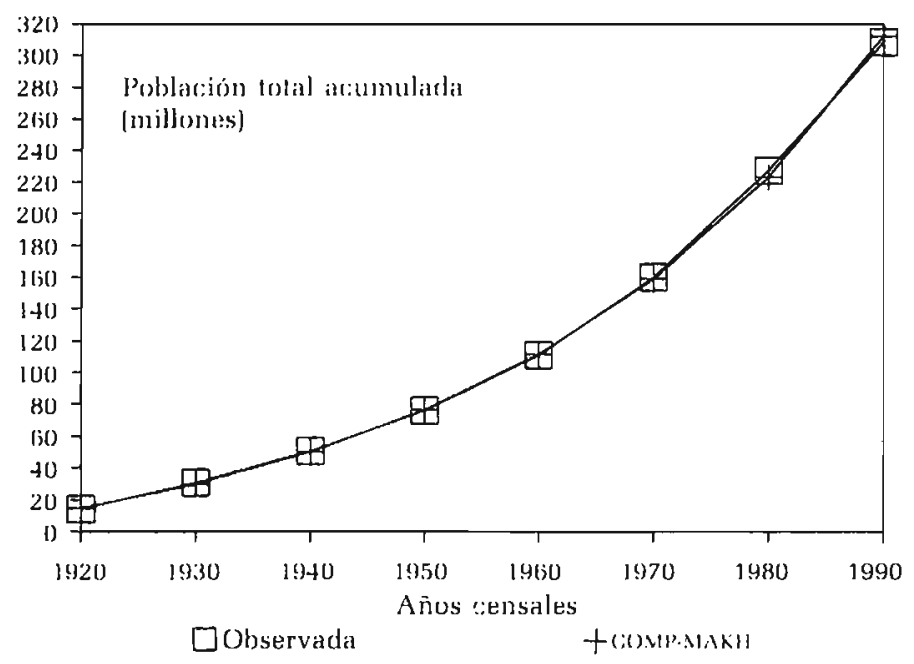

\section{GRÁFICA 2}

\section{México 1920-1990: crecimiento población}

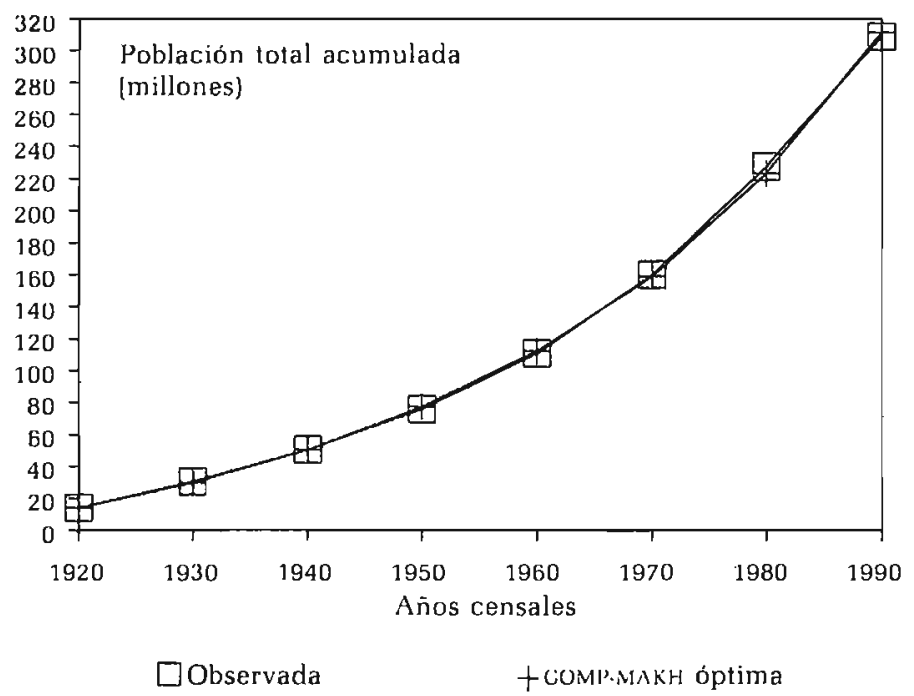


cedió a llevar éste al 30 de junio de 1920 y de esa forma los intervalos entre los datos se homogeneizaron a 10 años en todos los casos.

Con el método de los grupos no superpuestos se estimaron los parámetros de la función de Gompertz-Makeham y con ello se efectuó el ajuste descriptivo a los datos del crecimiento acumulado de población. El ajuste resultó magnífico, puesto que arrojó un coeficiente de determinación de 0.9996317 y una variación máxima al ajustar los valores estimados respecto a los valores observados de $-3.39 \%$ para 1930 [véase cuadro 2].

A su vez, la gráfica 1 corrobora cómo la función de GompertzMakeham describe adecuadamente el crecimiento de la población mexicana en el periodo de 1920 a 1990 , de tal forma que su comportamiento parece netamente exponencial.

Para visualizar la dinámica del crecimiento poblacional, con la función de Gompertz-Makeham se desagregó y obtuvo una estimáción de los valores de población total, para cada uno de los años censales (véase cuadro 3 ).

No obstante, a pesar de tener un ajuste excelente, se buscó precisar aún más la descripción del fenómeno, a través del método iterativo descrito en el capítulo de metodología, por lo que se generó una nueva función de Gompertz-Makeham que resultara óptima ${ }^{2}$ en su aplicación.

En el cuadro 4 y en la gráfica 2 se presentan los resultados alcanzados con la función de Gompertz-Makeham óptima. En ellos se aprecia que los valores estimados en la cuarta iteración mejoran la descripción inicial de los montos de crecimiento poblacional, dado que el coeficiente de determinación es ligeramente más alto que el anotado en el cuadro 2.

Ahora bien, en el cuadro 5, al comparar los parámetros "a" y " $k$ " de la función de Gompertz-Makeham óptima (cuarta iteración en este caso) se observa que prácticamente son iguales, mientras que los correspondientes a "d" $\mathrm{y}$ " $b$ " difieren ligeramente con los obtenidos inicialmente.

Así que al desagregar nuevamente con los parámetros de la función óptima (cuarta iteración), nos da como resultado los valores indicados en el cuadro 6; cifras que comparadas con las del cuadro 3 , suavizan ligeramente los valores estimados, sobre todo los correspondientes a 1930.

Con los datos del cuadro 6 se proyectaron al año 2000 y 2010 , por un lado los valores observados suponiendo un crecimiento ex-

\footnotetext{
${ }^{2}$ En el sentido de captar de la mejor manera posible el comportamiento del fenómeno en estudio.
} 
CUADRO 4

México 1920-1990: descripción del crecimiento poblacional acumulado mediante la función de Gompertz-Makeham óptima

\begin{tabular}{lccc}
\hline & \multicolumn{2}{c}{ Población acumulada } & $\begin{array}{c}\text { Variación } \\
\text { (porcentaje) }\end{array}$ \\
\cline { 2 - 3 } Año & observada & estimada & -0.57 \\
1920 & 13993424 & 13913661 & -2.34 \\
1930 & 30583502 & 29885750 & 0.77 \\
1940 & 50404767 & 50796920 & 1.57 \\
1950 & 76249358 & 77466185 & 0.98 \\
1960 & 111246230 & 112344590 & -0.59 \\
1970 & 160117797 & 159173765 & -1.78 \\
1980 & 227062782 & 223100105 & 0.79 \\
1990 & 308694622 & 311138470 & \\
& & & \\
\end{tabular}

ponencial a una tasa de crecimiento de la población de $1.5 \%$ en ésta y la siguiente década y por otro, los valores estimados aplicando la ley de Gompertz-Makeham (véase cuadro 7).

En la gráfica 3 se observan las curvas relativas a las proyecciones, una con la función aquí estudiada y la otra con la tendencia de crecimiento exponencial.

La discrepancia en los valores proyectados en las dos curvas parece a primera vista una contradicción, ya que si la función de Gompertz-Makeham, como hemos visto, describe adecuadamente el fenómeno en cuestión, es porque ha logrado asimilar su patrón de comportamiento y por tanto debiera poder predecir lo que sucederá en el futuro. Sin embargo, tal predicción sería válida siempre y cuando las condiciones no cambiaran.

Anteriormente se señaló que la fecundidad (actualmente componente fundamental en el crecimiento de la población en México) ha declinado marcadamente a partir de los setenta y es por eso que el comportamiento registrado en la gráfica 3 para el crecimiento de la población muestra un punto de inflexión, alrededor

CUADRO 5

México 1920-1990: estimación de parámetros de la función de GompertzMakeham

\begin{tabular}{ccc}
\hline & Valores iniciales & Cuarta iteración \\
\hline $\mathrm{K}$ & 31372780 & 31372506 \\
$\mathrm{a}$ & 1.38963 & 1.38859 \\
$\mathrm{~b}$ & 0.45845 & 0.44350 \\
$\mathrm{~d}$ & 0.49737 & 0.46351 \\
\hline
\end{tabular}


CUADRO 6

México 1920-1990: desagregación del crecimiento poblacional acumulado mediante la función de Gompertz-Makeham óptima

\begin{tabular}{llrc}
\hline & \multicolumn{2}{c}{ Población al 30 de junio } & $\begin{array}{c}\text { Variación } \\
\text { (porcentaje) }\end{array}$ \\
\cline { 2 - 3 } Año & observada & estimada & -0.57 \\
1920 & 13993424 & 13913661 & -3.87 \\
1930 & 16590078 & 15971474 & 5.21 \\
1940 & 19821266 & 20911786 & 3.09 \\
1950 & 25844590 & 26669265 & -0.34 \\
1960 & 34996872 & 34878405 & -4.36 \\
1970 & 48871567 & 46829175 & -4.72 \\
1980 & 66944985 & 63926340 & 7.28 \\
1990 & 81631840 & 88038365 & \\
\hline
\end{tabular}

de los años ochenta; es decir, hay un cambio en la concavidad de la curva, de tal forma que los puntos proyectados por la función de Gompertz-Makeham se "disparan".

La disminución del comportamiento registrado en la fecundidad ha sido tan radical, que en unos cuantos años se ha pasado, de acuerdo con las estimaciones del Consejo Nacional de Población (Conapo), de una tasa global de fecundidad (TGF) de casi 6.5 hijos por mujer en 1970 a una de sólo 3.16 en 1988.

Después de estimar una nueva función de Gompertz-Makeham, a fin de mejorar la proyección, se describió el crecimiento de la población en el periodo que va de 1960 a 1990, de tal manera que captara el momento de cambio en el ritmo de crecimiento y luego, con tal función, se proyectó al año 2000 y 2010, como se muestra en el cuadro 8 y en la gráfica 4.

Ahora el resultado es bastante congruente, al menos para el año 2000 , con lo que se espera a fin de siglo, con base en las cifras preliminares arrojadas por el Censo de 1990.

\section{CUADRO 7}

México 2000-2010: proyección del crecimiento poblacional exponencial vs. Gompertz-Makeham

\begin{tabular}{crc}
\hline & \multicolumn{2}{c}{$\begin{array}{c}\text { Población proyectada } \\
\text { Proyección }\end{array}$} \\
\cline { 2 - 3 } Año & exponencial & Gompertz-Makeham \\
\hline 2000 & 94842667 & 121773718 \\
2010 & 110191458 & 168786094 \\
\hline
\end{tabular}


GRÁFICA 3

México: proyección población 2000-2010

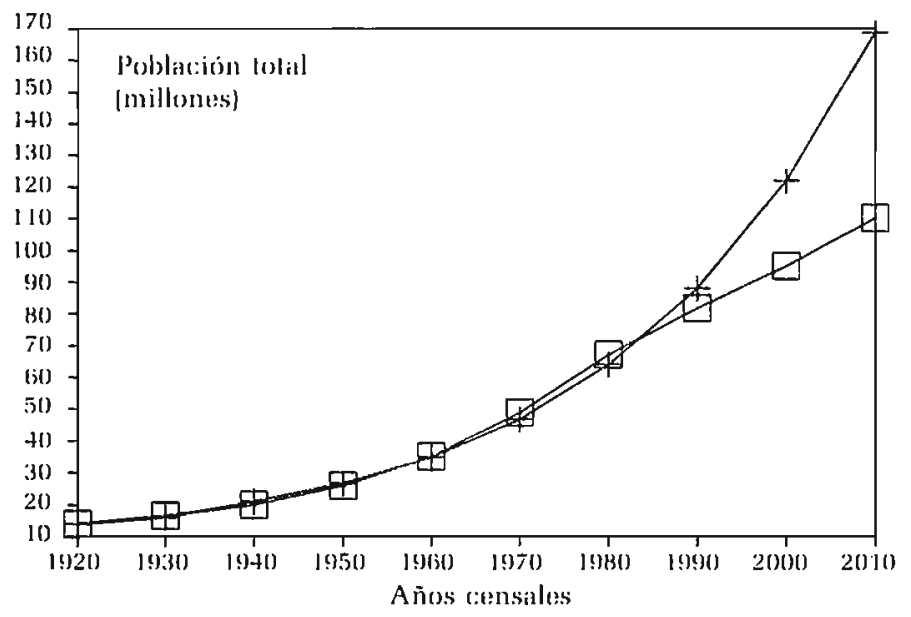

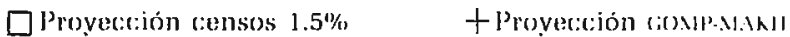

\section{GRÁFICA 4}

México: proyección población 2000-2010

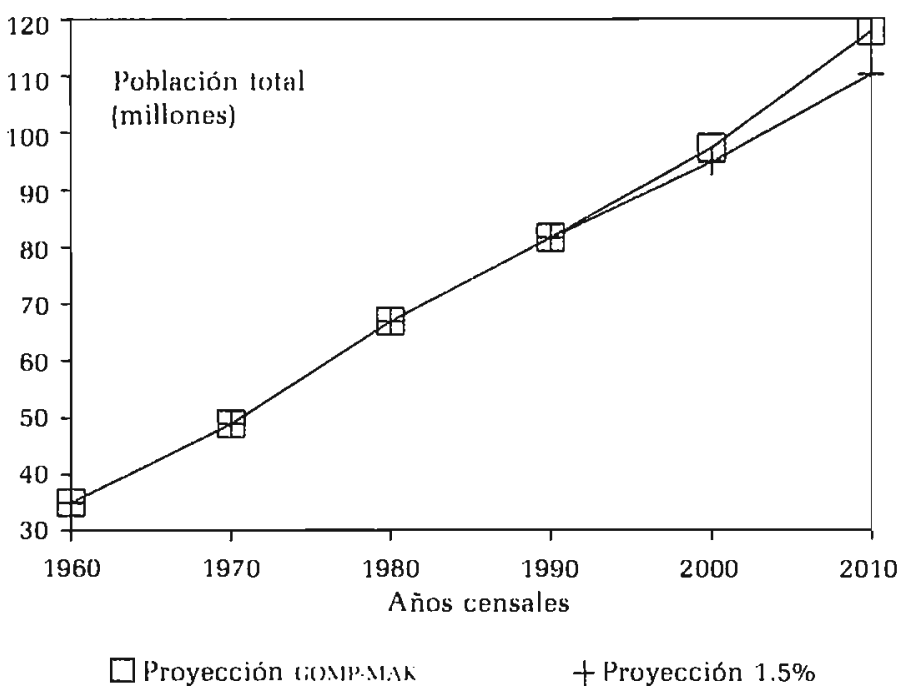


CUADRO 8

México 1960-2010: proyección del crecimiento poblacional mediante una nueva función de Gompertz-Makeham 1960-1990

\begin{tabular}{|c|c|c|}
\hline Año & Población proyectada & $\begin{array}{c}\text { Parámetros de } \\
\text { Gompertz-Makeham }\end{array}$ \\
\hline 1960 & 34996872 & \\
\hline 1970 & 48871567 & $K=133506131$ \\
\hline 1980 & 66944985 & 1.28903 \\
\hline 1990 & 81631840 & 0.26214 \\
\hline 2000 & 97356692 & 0.53685 \\
\hline 2010 & 117794675 & \\
\hline
\end{tabular}

Descripción de la estructura de la fecundidad por edades

El estado actual de la población en México está determinado por una revolución demográfica: al tipo tradicional de reproducción, caracterizado por altos índices de fecundidad y mortalidad, le sucede el tipo moderno, con tasas más bajas en ambos rubros.

Este hecho explica, como se señaló en el apartado anterior, el interés por los procesos y los pronósticos demográficos, así como el deseo de preveer las consecuencias ecológicas, económicas y sociales del crecimiento de la población.

Para caracterizar las actuales tendencias demográficas en nuestro país, es preciso prestar atención a la estructura de la fecundidad por edades, ${ }^{3}$ porque el crecimiento de la población depende principalmente de ello.

E] crecimiento de la población es la forma en que se manifiesta su reproducción, es decir, el relevo generacional. Por ejemplo, si durante un largo periodo de tiempo, a la generación de los padres le sucede una generación filial del mismo valor numérico, la población deja de crecer; y si además se mantiene invariable la esperanza de vida al nacimiento, también será permanente la estructura por edades. ${ }^{4}$

Cuando la generación de los hijos es mayor que la de los padres, la población crece; si es menor, al paso del tiempo, la población comenzará a decrecer inevitablemente.

$\mathrm{Si}$ la proporción de mujeres jóvenes es grande y relativamente baja la de ancianos (como en el caso de México), la población puede seguir creciendo, incluso considerablemente, aún en los casos

${ }^{3}$ La estructura de la fecundidad por edad se refiere a la distribución relativa de cómo las mujeres tienen a sus hijos.

${ }^{4} \mathrm{~A}$ una población de este tipo se le llama estacionaria. 
en que la fecundidad baje bastante y la reproducción no alcance a cubrir a la generación de los padres.

Sin embargo, como pasa en nuestro país, a una abundante generación de mujeres en edad procreativa, a través del tiempo, le sucederá otra menos numerosa, por lo que la población envejecerá a un ritmo que se irá acelerando, a la par de un aumento en la tasa general de mortalidad.

La fecundidad como componente demográfico se manifiesta esencialmente a través de la estructura por edad, es decir, por las tasas especificas de fecundidad por edades y, desde luego, por su nivel medido por la suma de tales tasas, conocida como la tasa global de fecundidad (TGF).

Por tanto, describir la estructura de la fecundidad por edades resulta importante para la formulación de políticas de población y en particular de los programas de planificación familiar, ya que lo que cuenta para la reproducción y la exposición al riesgo de concebir depende de la proporción de mujeres en edad reproductiva.

Asimismo, esta descripción sirve como marco de referencia para el análisis de la práctica anticonceptiva y por tanto, ello permite determinar, de los diferentes grupos de edad, cuáles han sido los principales responsables de los cambios ocurridos en la fecundidad.

A] aplicar la función de Gompertz-Makeham para describir la estructura de la fecundidad por edad se consideraron las estimaciones de las tasas específicas correspondientes para los años de 1952, 1970 y 1988 que aparecen en el México Demográfico -breviario de 1988- publicado por Conapo.

En un primer momento, la descripción efectuada con la función de Gompertz-Makeham parecía poco apropiada (véase cuadro 9 y gráfica 5), porque con el ajuste se modificó la composición relativa de la estructura por edad; es decir, la aportación del grupo 20-24 disminuyó, mientras que la del grupo 25-29 aumentó; situación que no resulta muy aceptable para 1988 , porque a una fecundidad que tiende a la baja, pasando la transición, debiera corresponderle una cúspide temprana.

Por ello se efectuó un ajuste correctivo de la estructura por edad con el método bilogístico, suponiendo que la proporción de la fecundidad global experimentada por una población hasta la edad $x$, sigue una distribución Gompertz. ${ }^{5}$

Al aplicar el método bilogístico, cambió la forma de la estruc-

\footnotetext{
${ }^{5}$ Para ver los detalles del método bilogístico se remite al Manual $X$ de la o:l. Técnicus Indirectus de Estimución Demográficu.
} 
CUADRO 9

México: descripción de la estructura de la fecundidad por edad

\begin{tabular}{|c|c|c|c|c|c|c|}
\hline \multirow{3}{*}{$\begin{array}{c}\text { Grupos } \\
\text { edad }\end{array}$} & \multicolumn{6}{|c|}{ Tasas específicas } \\
\hline & \multicolumn{2}{|c|}{1952} & \multicolumn{2}{|c|}{1970} & \multicolumn{2}{|c|}{1988} \\
\hline & observada & estimada & observada & estimada & observada & estimada \\
\hline $15-19$ & 0.1200 & 0.0611 & 0.0913 & 0.0510 & 0.0553 & 0.0262 \\
\hline $20-24$ & 0.3020 & 0.2815 & 0.2839 & 0.2551 & 0.1698 & 0.1384 \\
\hline $25-29$ & 0.3135 & 0.3626 & 0.3140 & 0.3579 & 0.1632 & 0.2132 \\
\hline $30-34$ & 0.2586 & 0.2739 & 0.2470 & 0.2890 & 0.1235 & 0.1539 \\
\hline $35-39$ & 0.1625 & 0.1654 & 0.2097 & 0.1836 & 0.0814 & 0.0696 \\
\hline $40-44$ & 0.0637 & 0.0910 & 0.0767 & 0.1050 & 0.0345 & 0.0236 \\
\hline $45-49$ & 0.0637 & 0.0481 & 0.0767 & 0.0574 & 0.0043 & 0.0067 \\
\hline TGF & 6.4 & 6.4 & 6.5 & 6.5 & 3.2 & 3.2 \\
\hline \multirow[t]{5}{*}{ Coef. de } & e Determ.: & 0.9267 & & 0.9178 & & 0.8673 \\
\hline & \multicolumn{6}{|c|}{ Edad media a la fecundidad en años } \\
\hline & \multicolumn{2}{|c|}{1952} & \multicolumn{2}{|c|}{1970} & \multicolumn{2}{|c|}{1988} \\
\hline & observada & estimada & observada & estimada & observada & estimada \\
\hline & 29.40 & 30.10 & 30.34 & 30.75 & 28.50 & 29.08 \\
\hline
\end{tabular}

tura por edad, de cúspide tardía a temprana para los años de 1952 y 1970 , debido al patrón estándar utilizado para el ajuste, el cual tiende a imponer su perfil. El interés por tener una estructura corregida era que al describirla nuevamente con la función de

CUADRO 10

México: ajuste de la estructura por edad de la fecundidad por el método bilogístico

\begin{tabular}{|c|c|c|c|c|c|c|}
\hline \multirow{3}{*}{$\begin{array}{c}\text { Grupos } \\
\text { edad }\end{array}$} & \multicolumn{6}{|c|}{ Tasas específicas } \\
\hline & \multicolumn{2}{|c|}{1952} & \multicolumn{2}{|c|}{1970} & \multicolumn{2}{|c|}{1988} \\
\hline & observada & ajustada & observada & ajustada & observada & ajustada \\
\hline $15-19$ & 0.1200 & 0.0728 & 0.0913 & 0.0574 & 0.0553 & 0.0145 \\
\hline $20-24$ & 0.3020 & 0.3943 & 0.2839 & 0.3572 & 0.1698 & 0.2393 \\
\hline $25-29$ & 0.3135 & 0.3066 & 0.3140 & 0.3078 & 0.1632 & 0.1888 \\
\hline $30-34$ & 0.2586 & 0.2120 & 0.2470 & 0.2269 & 0.1235 & 0.1029 \\
\hline $35-39$ & 0.1625 & 0.1477 & 0.2097 & 0.1664 & 0.0814 & 0.0538 \\
\hline $40-44$ & 0.0637 & 0.0987 & 0.0767 & $0.1^{\prime} 171$ & 0.0345 & 0.0252 \\
\hline $45-49$ & 0.0637 & 0.0519 & 0.0767 & 0.0664 & 0.0043 & 0.0074 \\
\hline $\mathrm{TGF}$ & 6.4 & 6.4 & 6.5 & 6.5 & 3.2 & 3.2 \\
\hline
\end{tabular}


Gompertz-Makeham, no se sesgara la estimación del aporte a la fecundidad hecha por los grupos de 20-24 y 25-29 años de edad, como había sucedido inicialmente (véase cuadro 10).

Una vez corregida la estructura por edad, se le aplicó la función de Gompertz-Makeham y los valöres estimados para 1988 reprodujeron aceptablemente dicha estructura, observada con una correlación mucho más alta que la mostrada en el cuadro 9. La descripción de los años de 1952 y 1970 arrojó, en la estructura por edad, una cúspide temprana y un coeficiente de determinación ligeramente menor que en la primera aplicación de la función, debido a las características del patrón estándar utilizado en el método bilogístico (véase cuadro 11 y gráfica 6).

Para elegir lo que podrian ser las mejores descripciones, ${ }^{6}$ se consideró el nivel de correlación medido por el coeficiente de determinación y la forma que guarda la estructura por edad de acuerdo con las condiciones de la fecundidad en el momento de estudio. Es decir, mientras menor sea el nivel de la fecundidad, la estructura se caracteriza por un peso mayor de la misma a edades más jóvenes e implica, entonces, una disminución en la edad media de la ocurrencia del fenómeno.

Por tanto, para 1952 y 1970 el primer ajuste descriptivo (cuadro 9) era el más adecuado, por sus coeficientes de determinación superiores al otro ajuste y porque reproduce mejor la forma de la estructura (cúspide tardía para periodos de fecundidad alta). A su vez, para 1988 el segundo ajuste descriptivo (corregida la estructura), era el más adecuado, por su coeficiente de determinación mayor que el ajuste anterior y por dar la conformación apropiada (cúspide temprana a fecundidad baja) con una edad media a la fecundidad tendiente a la baja.

Con fines de proyección de la estructura por edad de la fecundidad, generalmente se proyecta la tasa global de fecundidad por un lado, y la estructura porcentual de las tasas específicas por otro, contando a su vez con alguna hipótesis futura del nivel y con alguna distribución meta para los periodos de proyección.

En esta ocasión se prefirió observar la tendencia seguida por los parámetros de la función de Gompertz-Makeham para los tres años en estudio (véase cuadro 12).

Al aumento en los niveles de fecundidad de 1952 a 1970 , los parámetros "a" y "d" estuvieron al alza, mientras que " $b$ " y " $K$ " fueron a la baja. En cambio, al disminuir la fecundidad en el pe-

\footnotetext{
6 También aquí se buscó una función de Gompartz-Makeham óptima a través de] método iterativo; sin embargo, para los tres años considerados no se alcanzó convergencia en los valores de los parámetros al paso de las iteracciones.
} 
GRÁFICA 5

México: estructura fecundidad $x$ edad

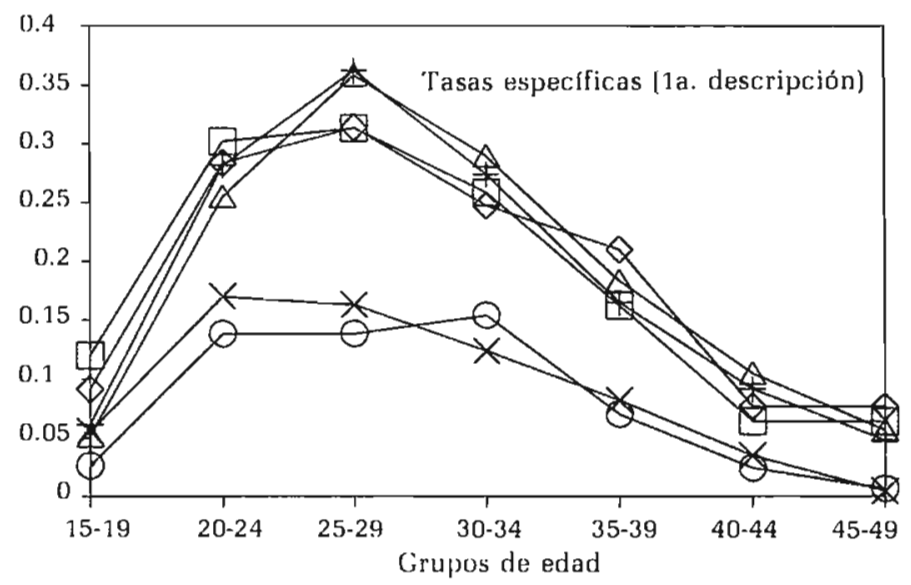

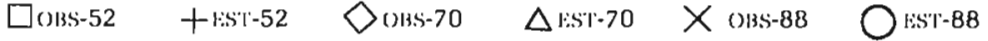

GRÁFICA 6

México: estructura fecundidad $x$ edad

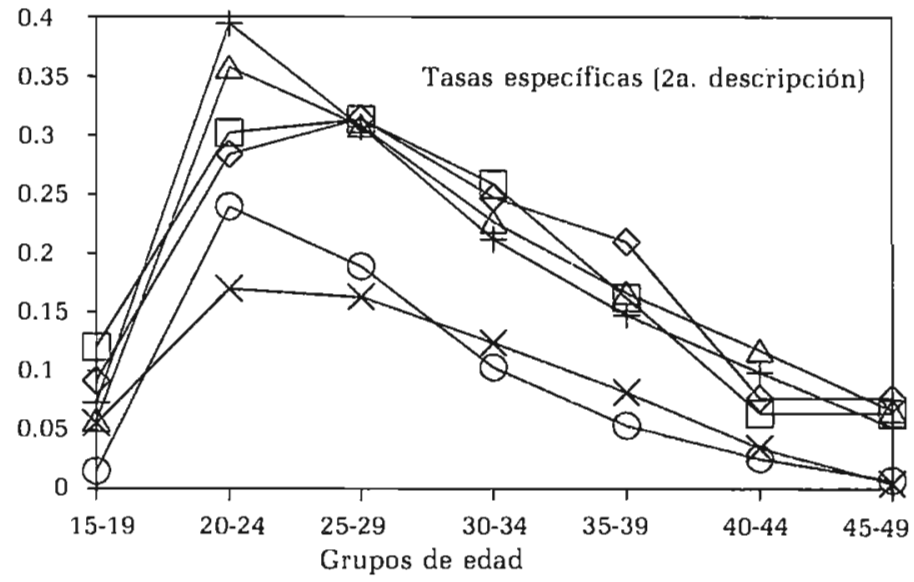

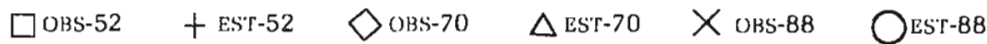


CUADRO 11

México: descripción de la estructura corregida por edad de la Fecundidad

\begin{tabular}{|c|c|c|c|c|c|c|}
\hline \multirow{3}{*}{$\begin{array}{c}\text { Grupos } \\
\text { edad }\end{array}$} & \multicolumn{6}{|c|}{ Tasas especificas } \\
\hline & \multicolumn{2}{|c|}{1952} & \multicolumn{2}{|c|}{1970} & \multicolumn{2}{|c|}{1988} \\
\hline & observada & estimada & observada & estimada & observada & estimada \\
\hline $15-19$ & 0.1200 & 0.1236 & 0.0913 & 0.1020 & 0.0553 & 0.0456 \\
\hline $20-24$ & 0.3020 & 0.3573 & 0.2839 & 0.3276 & 0.1698 & 0.2099 \\
\hline $25-29$ & 0.3135 & 0.3131 & 0.3140 & 0.3135 & 0.1632 & 0.1951 \\
\hline $30-34$ & 0.2586 & 0.2124 & 0.2470 & 0.2275 & 0.1235 & 0.1056 \\
\hline $35-39$ & 0.1625 & 0.1364 & 0.2097 & 0.1550 & 0.0814 & 0.0476 \\
\hline $40-44$ & 0.0637 & 0.0865 & 0.0767 & 0.1041 & 0.0345 & 0.0201 \\
\hline $45-49$ & 0.0637 & 0.0548 & 0.0767 & 0.0697 & 0.0043 & 0.0084 \\
\hline TGF & 6.4 & 6.4 & 6.5 & 6.5 & 3.2 & 3.2 \\
\hline \multirow[t]{5}{*}{ Coef. de } & e Determ.: & 0.9190 & & 0.9071 & & 0.9283 \\
\hline & \multicolumn{6}{|c|}{ Edad media a la fecundidad en años } \\
\hline & \multicolumn{2}{|c|}{1952} & \multicolumn{2}{|c|}{1970} & \multicolumn{2}{|c|}{1988} \\
\hline & observada & $\overline{\text { estimada }}$ & observada & $\overline{\text { estimada }}$ & observada & $\overline{\text { estimada }}$ \\
\hline & 29.40 & 28.90 & 30.34 & 29.80 & 28.50 & 27.45 \\
\hline
\end{tabular}

riodo de 1970 a 1988 , la relación muestra un camino descendente en todo momento para todos los parámetros.

Con base en tales tendencias (en especial con la baja en la fecundidad), se simularon lo que podría ser en el futuro próximo las estructuras por edad de la fecundidad, a través de variar los valores de los parámetros de la función de Gompertz-Makeham, buscando congruencia con la tendencia manifestada (véase cuadro 13 y gráficas 7 y 8 ].

De las alternativas resultantes, bien se puede tomar la que dis-

CUADRO 12

México: descripción de la estructura de la fecundidad por edad.

Parámetros de la función Gompertz-Makeham estimados con el método de los grupos no superpuestos

\begin{tabular}{cccc}
\hline Parámetros & 1952 & 1970 & 1988 \\
\hline$K=$ & 4.946926 & 4.920333 & 4.445418 \\
$\mathrm{a}=$ & 0.513957 & 0.527822 & 0.410384 \\
$\mathrm{~b}=$ & 0.000123 & 0.000106 & 0.000020 \\
$\mathrm{~d}=$ & 0.419032 & 0.434887 & 0.338064 \\
\hline
\end{tabular}


minuye en $7.5 \%$ el valor de los parámetros, como la posible estructura de la fecundidad por edad para el año 2000, supuesta como meta a alcanzar una tasa global de fecundidad de 2.7 hijos por mujer (véase gráfica 7).

En cuanto a la distribución porcentual de las estructuras de fecundidad por edad mostradas en el cuadro 13 y en la gráfica 8 se observa que conforme la fecundidad continúe su descenso, la participación de las mujeres en la procreación se incrementará en los grupos de edad más jóvenes, como son el de 15-19 y 20-24; mientras que disminuirá ligeramente en los grupos de edad de 2529 y $30-34$ y tendrá un marcado descenso para los grupos de mayor edad [35-49 años].

Cualquiera de las alternativas que se considere, a excepción de las que disminuyen en $1 \%$ y $2.5 \%$ el valor de los parámetros de la función de Gompertz-Makeham, en los grupos de edad de 35 a 49 años, el aporte de estas mujeres a la fecundidad total del país estará por debajo de 10 por ciento.

\section{Descripción de la estructura por edad de la PEA ocupada}

Como se apuntó anteriormente, en México la fecundidad es el aspecto demográfico que mayormente ha influido en el tamaño y la estructura por edad de la población; de ahf lo impresionante que ha sido el crecimiento y rejuvenecimiento de la población.

Este proceso puede visualizarse en dos periodos. El primero se observa desde los cuarenta hasta principios de los setenta, cuando la fecundidad se mantuvo alta y constante mientras la mortalidad disminufa considerablemente. El segundo se manifiesta desde principios de los setenta hasta el momento actual, con un marcado descenso en la fecundidad y el mantenimiento de tasas de mortalidad bajas; lo cual trae como consecuencia una sensible desaceleración en el ritmo de crecimiento de la población, que no obstante continúa creciendo por la inercia que guarda la estructura por edad generada en el primer periodo.

La población económicamente activa (PEA) está constituida por el grupo social que rebase los 12 años de edad, que año con año se incorpora al mercado de trabajo. En México, dada la estructura por edad actual de la población, la PEA aumenta a una tasa superior a la del crecimiento de la población total. Este hecho obliga a la reflexión sobre el intenso incremento de la demanda de empleos que se perfila sobre todo entre los jóvenes para el presente y hacia los próximos años. Respecto a la oferta, hay que considerar por un lado que los empleos deberán ser urbanos y compe- 
CUADRO 13

México: descripción de la estructura de la fecundidad por edad

\begin{tabular}{llllllr}
\hline & \multicolumn{5}{c}{ Tasas específicas } \\
\cline { 2 - 7 } $\begin{array}{c}\text { Grupos } \\
\text { edad }\end{array}$ & \multicolumn{6}{c}{ Disminución en pàrámetros de la función G-M } \\
\cline { 2 - 7 } & 1988 & $10 \%$ & $\mathbf{7 . 5 \%}$ & $\mathbf{5 \%} \%$ & $\mathbf{2 . 5 \%}$ & $1 \%$ \\
\hline $15-19$ & 0.0472 & 0.0534 & 0.0519 & 0.0504 & 0.0488 & 0.0479 \\
$20-24$ & 0.2177 & 0.1987 & 0.2044 & 0.2094 & 0.2139 & 0.2163 \\
$25-29$ & 0.2024 & 0.1482 & 0.1612 & 0.1746 & 0.1884 & 0.1967 \\
$30-34$ & 0.1095 & 0.0678 & 0.0769 & 0.0869 & 0.0978 & 0.1047 \\
$35-39$ & 0.0493 & 0.0267 & 0.0314 & 0.0367 & 0.0426 & 0.0466 \\
$40-44$ & 0.0209 & 0.0101 & 0.0122 & 0.0147 & 0.0175 & 0.0195 \\
$45-49$ & 0.0087 & 0.0037 & 0.0047 & 0.0058 & 0.0071 & 0.0080 \\
& & & & & & \\
TCF & 3.3 & 2.5 & 2.7 & 2.9 & 3.1 & 3.2 \\
\hline
\end{tabular}

México: distribución porcentual de la estructura por edad de la fecundidad

\begin{tabular}{|c|c|c|c|c|c|c|}
\hline \multirow{2}{*}{$\begin{array}{c}\text { Grupos } \\
\text { edad }\end{array}$} & \multirow[b]{2}{*}{1988} & \multicolumn{5}{|c|}{ Disminución en parámetros de la función G-M } \\
\hline & & $10 \%$ & $7.5 \%$ & $5 \%$ & $2.5 \%$ & $1 \%$ \\
\hline $15-19$ & 7.21 & 10.50 & 9.56 & 8.70 & 7.92 & 7.48 \\
\hline $20-24$ & 33.20 & 39.07 & 37.66 & 36.21 & 34.72 & 33.81 \\
\hline $25-29$ & 30.86 & 29.14 & 29.71 & 30.19 & 30.58 & 30.76 \\
\hline $30-34$ & 16.70 & 13.33 & 14.18 & 15.03 & 15.87 & 16.37 \\
\hline $35-39$ & 7.52 & 5.25 & 5.78 & 6.34 & 6.92 & 7.28 \\
\hline $40-44$ & 3.19 & 1.98 & 2.24 & 2.53 & 2.85 & 3.05 \\
\hline $45-49$ & 1.32 & 0.74 & 0.86 & 1.00 & 1.15 & 1.25 \\
\hline \multirow{2}{*}{$\begin{array}{c}\text { Grupos } \\
\text { edad }\end{array}$} & & \multicolumn{5}{|c|}{ Disminución en parámetros de la función $G-M$} \\
\hline & 1988 & $10 \%$ & $7.5 \%$ & $5 \%$ & $2.5 \%$ & $1 \%$ \\
\hline $15-34$ & 87.97 & 92.03 & 91.12 & 90.13 & 89.09 & 88.42 \\
\hline $35-49$ & 12.03 & 7.97 & 8.88 & 9.87 & 10.91 & 11.58 \\
\hline
\end{tabular}

titivos, y por otro que la creación de éstos implica la penosa necesidad de destruir los empleos ineficientes que merman la productividad y la competitividad.

Desde luego cabe esperar que mientras la fecundidad continúe su reducción, se registrará un envejecimiento creciente que afectara a toda la estructura por edad de la población. Mientras ello sucede, el ritmo de crecimiento de la PEA seguirá sobrepasando al del crecimiento de la población con el consecuente impacto en la estructura económica y social del país.

De ahí la necesidad de lograr una descripción adecuada de la estructura por edad de la PEA, para estar en posibilidad de abor- 
GRÁFICA 7

México: proyección estructuras fecundidad

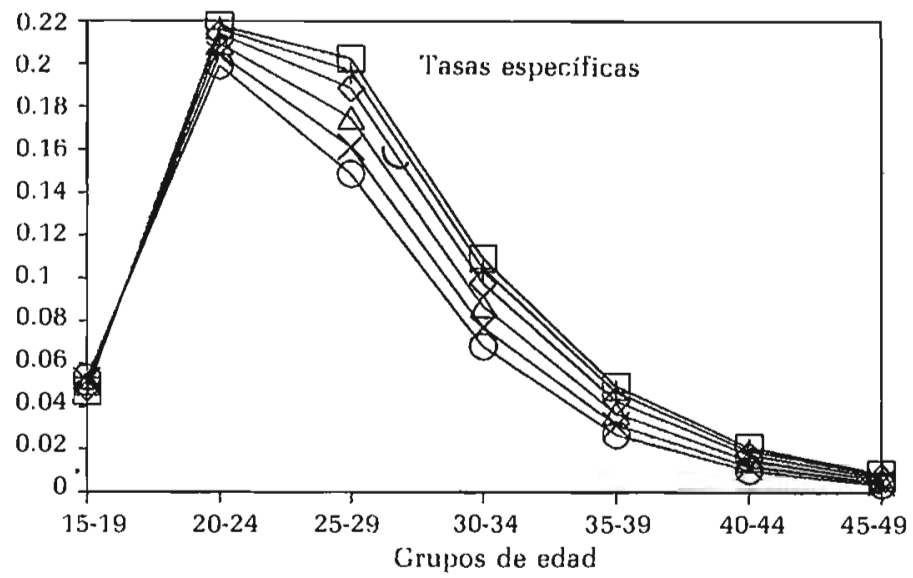

$\square 1988 \quad+1 \% \quad \diamond 2.5 \% \quad \Delta 5 \% \quad \times 7.5 \% \quad \bigcirc 10 \%$

GRÁFICA 8

México: distribución \% estructuras fecundidad

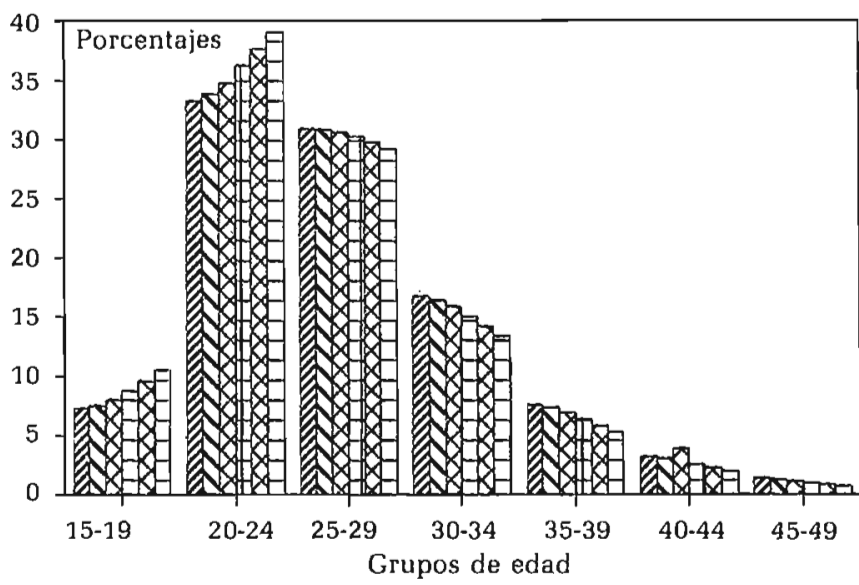

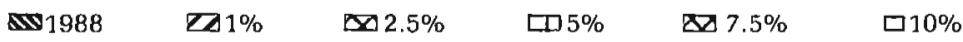


dar y dar respuestas a la problemática que significa la creciente demanda de empleos.

En una economía como la nuestra, donde existe un gran número de personas activas que participan marginalmente en el mercado de trabajo o simplemente permanecen desocupadas, la estimación precisa de la PEA se dificulta. Por consiguiente pensando en los fines de este trabajo, para la descripción de la estructura por edad de la PEA se tomaron los datos correspondientes a la PEA ocupada total de México, cuyos valores fueron emitidos por la Encuesta Complementaria a la Encuesta Continua sobre Ocupación, elaborada por la SPP. La información corresponde al periodo comprendido entre el 11 de octubre de 1976 y el 7 de enero de 1977, es decir, al último trimestre de 1976.

La aplicación de la función de Gompertz-Makeham para describir la estructura por edad de la PEA ocupada, consistió, en primer lugar, en estimar los parámetros de dicha función matemática con el método de los grupos no superpuestos y en segundo lugar, en efectuar el ajuste descriptivo.

En el cuadro 14 y en la gráfica 9 se observa que la descripción mediante la función de Gompertz-Makeham de la estructura por edad de la PEA ocupada acumulada resultó estupenda, con un coeficiente de determinación de 0.9981867 y con alguna variación en los primeros grupos de edad, para después empatar prácticamente los valores observados y estimados.

También en el cuadro 15 se presenta el resultado del ajuste descriptivo expresado en números relativos, donde se comprueba que los datos observados y los estimados son muy semejantes.

A su vez, para captar el estado que guarda la estructura por edad individual de la PEA ocupada, con la misma función de Gompertz-Makeham se realizó la desagregación de los grupos quinquenales. Esta situación se presenta en el cuadro 16 y en la gráfica 10.

Es significativa la figura resultante de la gráfica 10 porque es típica de] comportamiento por edad de la PEA: la población ocupada tiene una participación máxima en la economía del país en las edades comprendidas entre los 19 y los 35 años. Para este año en particular, el punto máximo correspondió a la edad de 26 años, promedio representativo de una población joven. A partir de ahí, muestra una declinación muy suavizada y en las últimas edades el comportamiento tiende a ser constante.

Se buscó una función de Gompertz-Makeham óptima, pero no se alcanzó convergencia en los valores de los parámetros, debido a que desde la primera aplicación descriptiva la correlación para este caso es muy alta. 
CUADRO 14

México 1976: descripción de la estructura por edad de la PEA ocupada acumulada a través de la función de Gompertz-Makeham

\begin{tabular}{cccc}
\hline $\begin{array}{c}\text { Grupo de } \\
\text { edad }\end{array}$ & \multicolumn{2}{c}{ PEA ocupada acumulada } & $\begin{array}{c}\text { Variación } \\
\text { [porcentaje) }\end{array}$ \\
\cline { 2 - 3 } $12-14$ & observada & estimada & 37.46 \\
$15-19$ & 168935 & 232220 & -16.65 \\
$20-24$ & 259988 & 995154 & -12.40 \\
$25-29$ & 3787119 & 2276538 & -3.20 \\
$30-34$ & 4776131 & 4666005 & 1.57 \\
$35-39$ & 5634883 & 5751609 & 2.07 \\
$40-44$ & 6343240 & 6413081 & 1.10 \\
$45-49$ & 6907131 & 6909160 & 0.03 \\
$50-54$ & 7358221 & 7301965 & -0.76 \\
$55-59$ & 7678485 & 7634622 & -0.57 \\
$60-64$ & 7905983 & 7934634 & 0.36 \\
$65-69$ & 8172117 & 8218701 & 0.57 \\
& Coeficiente de determinación: 0.9981867 & \\
\hline
\end{tabular}

CUADRO 15

México 1976: descripción relativa de la estructura por edad de la PEA ocupada acumulada a través de la función de Gompertz-Makeham

\begin{tabular}{lcc}
\hline \multirow{2}{*}{$\begin{array}{c}\text { Grupo de } \\
\text { edad }\end{array}$} & \multicolumn{2}{c}{ PEA ocupada acumulada } \\
\cline { 2 - 3 } & $\begin{array}{c}\text { observada } \\
\text { (porcentaje) }\end{array}$ & $\begin{array}{c}\text { estimada } \\
\text { (porcentaje) }\end{array}$ \\
\hline $12-14$ & 2.07 & 2.83 \\
$15-19$ & 14.61 & 12.11 \\
$20-24$ & 31.80 & 27.70 \\
$25-29$ & 46.34 & 44.61 \\
$30-34$ & 58.44 & 59.02 \\
$35-39$ & 68.95 & 69.98 \\
$40-44$ & 77.62 & 78.03 \\
$45-49$ & 84.52 & 84.07 \\
$50-54$ & 90.04 & 88.85 \\
$55-59$ & 93.96 & 92.89 \\
$60-64$ & 96.74 & 96.54 \\
$65-69$ & 100.00 & 100.00 \\
\hline
\end{tabular}


GRÁFICA 9

México 1976: PEA ocupada acumulada

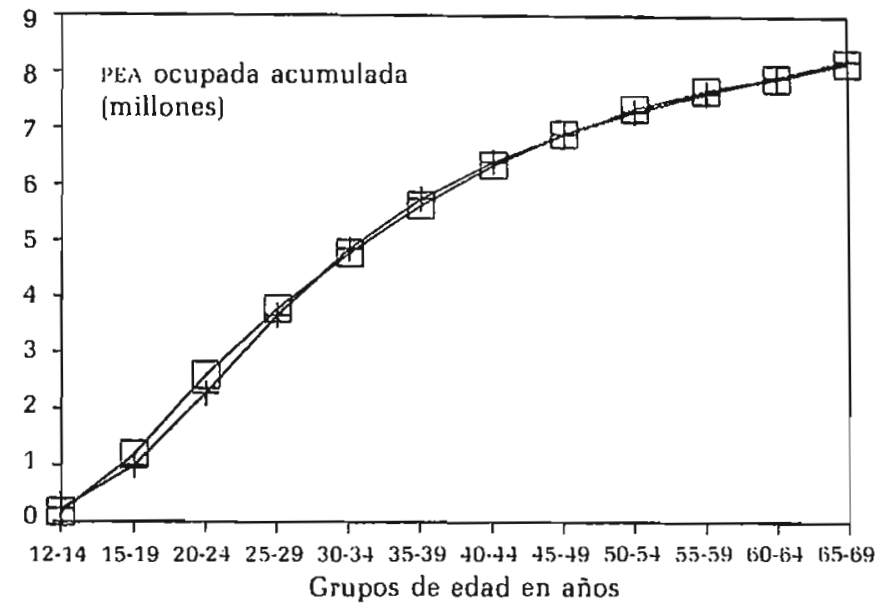

DObservada

+Estimada

\section{GRÁFICA 10}

México 1976: PEA ocupada por edad

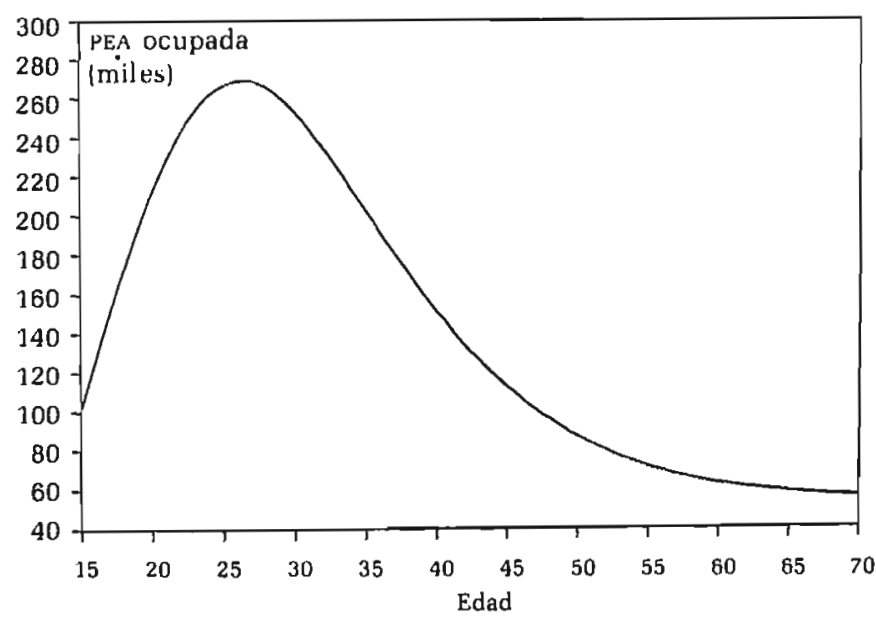


CUADRO 16

México 1976: desagregación de la PEA ocupada acumulada por edad individual cumplida a través de la función de Gompertz-Makeham

\begin{tabular}{cccccc}
\hline Edad & PEA & Edad & PEA & Edad & PEA \\
\hline 15 & 100885 & 34 & 214178 & 53 & 75268 \\
16 & 126481 & 35 & 202417 & 54 & 72627 \\
17 & 152920 & 36 & 190860 & 55 & 70271 \\
18 & 179013 & 37 & 179655 & 56 & 68176 \\
19 & 203635 & 38 & 168908 & 57 & 66316 \\
20 & 225815 & 39 & 158697 & 58 & 64672 \\
21 & 244812 & 40 & 149072 & 59 & 63222 \\
22 & 260138 & 41 & 140060 & 60 & 61948 \\
23 & 271558 & 42 & 131674 & 61 & 60835 \\
24 & 279061 & 43 & 123910 & 62 & 59866 \\
25 & 282823 & 44 & 116755 & 63 & 59027 \\
26 & 283155 & 45 & 110189 & 64 & 58306 \\
27 & 280462 & 46 & 104186 & 65 & 57692 \\
28 & 275197 & 47 & 98715 & 66 & 57173 \\
29 & 267831 & 48 & 93745 & 67 & 56741 \\
30 & 258819 & 49 & 89244 & 68 & 56387 \\
31 & 248588 & 50 & 85177 & 69 & 56104 \\
32 & 237523 & 51 & 81513 & & \\
33 & 225959 & 52 & 78220 & & \\
\hline
\end{tabular}

A fin de tener una idea del comportamiento probable en "diferentes escenarios" de la estructura por edad de la PEA ocupada, se procedió a variar en $+y-10 \%$ el valor de cada uno de los parámetros de la función de Gompertz-Makeham, manteniendo constante el valor de los otros.

Las diferentes opciones de la simulación dieron como resultado lo siguiente:

a) Al variar el parámetro " $K$ ", en la gráfica 11 se observa cómo se establece arriba y abajo una franja de valores más o menos equidistantes, tanto de los valores como de los estimados en la descripción inicial con la función de Gompertz-Makeham.

b) Con la variación del parámetro "a", la fluctuación en la estimación es enorme, por lo que en la descripción de la estructura por edad de la PEA ocupada en 1976 resultó muy sensible a los cambios de valor del parámetro "a" (véase gráfica 12).

c) En cuanto al parámetro "b", su disminución o incremento no altera para nada el ajuste descriptivo de la función de Gompertz-Makeham (véase gráfica 13).

d) En lo que respecta a los cambios en el parámetro "d", el ajuste descriptivo arrojó poca variación en las primeras y últimas 
GRÁFICA 11

México 1976: PEA, simulación param. $k$

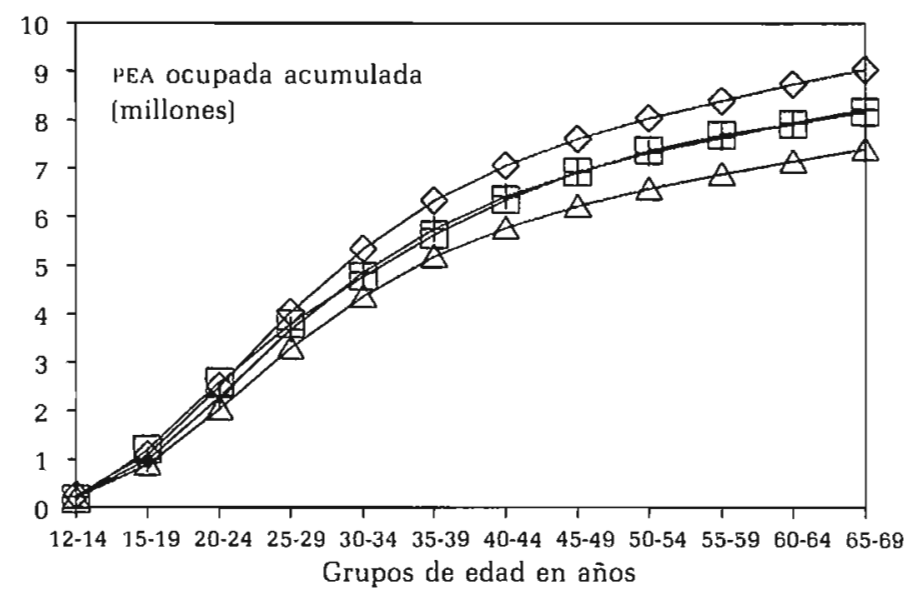

$\square$ oBs. $\quad$ +ЕST. $\quad \diamond \mathrm{k}(+10 \%) \quad \Delta \mathrm{k}(-10 \%)$

GRÁFICA 12

México 1976: PEA, simulación param. a

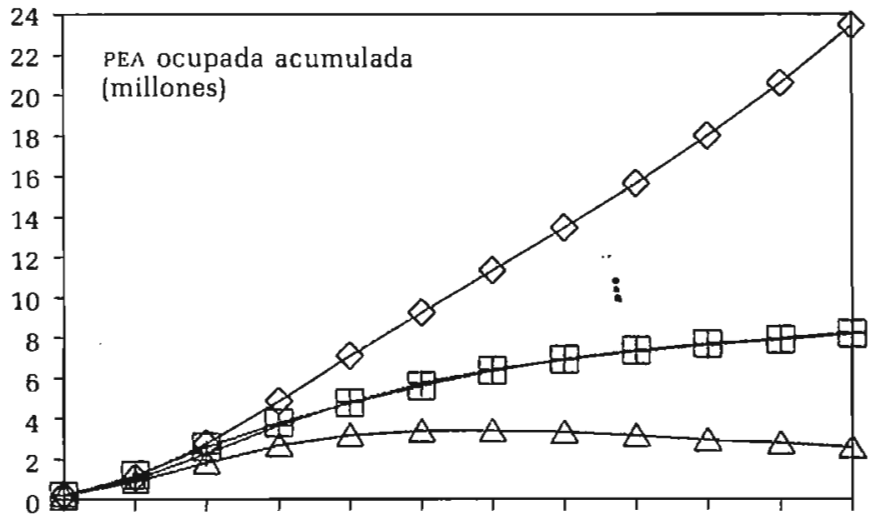

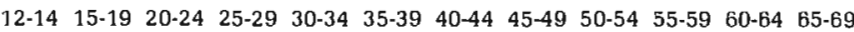
Grupos de edad en años
$\square$ ors.
+ EST.
$\diamond \mathrm{a}(+10 \%)$
$\triangle \mathrm{a}(-10 \%)$ 
DESCRIPCIÓN Y PROYECCIÓN DE FENÓMENOS DEMOGRÁFICOS

517

GRÁFICA 13

México 1976: PEA, simulación param. b

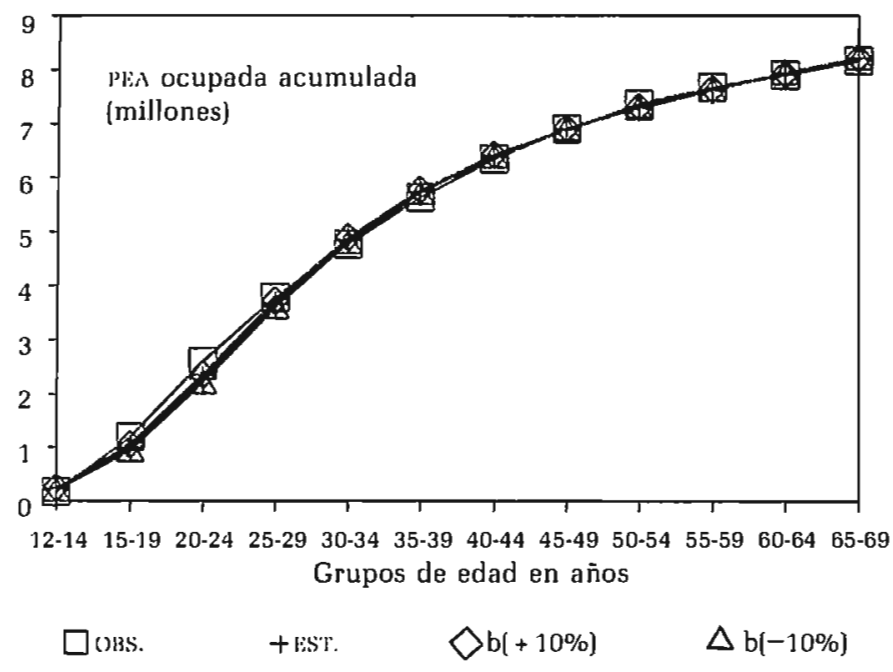

GRÁFICA 14

México 1976: PEA, simulación param. d

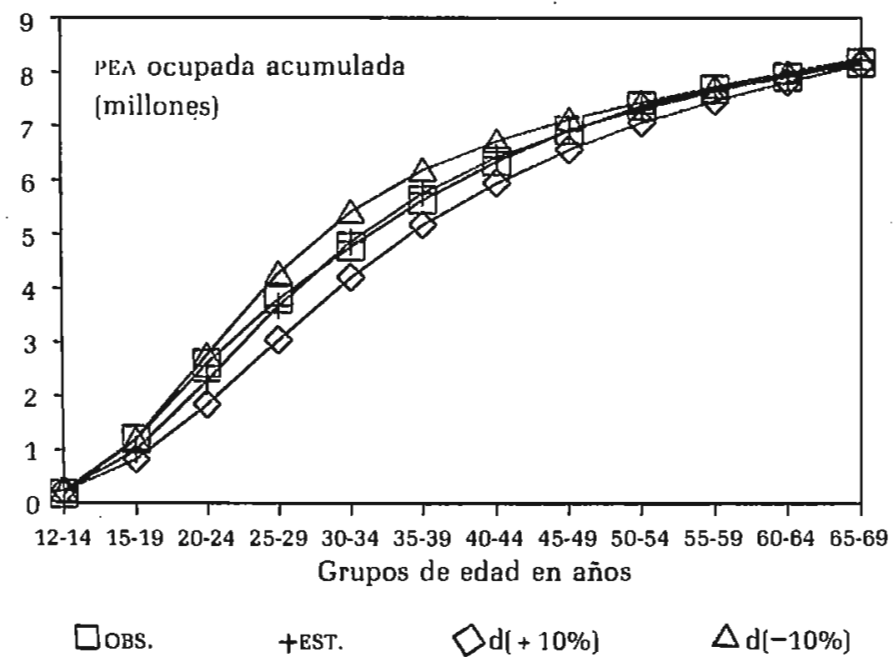


edades, pero mostrando mayor sensibilidad en los grupos de edad intermedios (véase gráfica 14).

\section{Conclusiones}

De acuerdo con los resultados obtenidos en el presente trabajo se comprueba que la función de Gompertz-Makeham es un modelo matemático que describe adecuadamente los fenómenos demográficos aquí estudiados.

El cálculo de los parámetros por el método de los grupos no superpuestos viene siendo un procedimiento sencillo de estimación. A su vez, el método iterativo empleado para encontrar funciones de Gompertz-Makeham óptimas fue muy útil para la aplicación relativa al crecimiento poblacional, ya que en algunas de las iteraciones se obtuvieron funciones que lograban una mejor descripción del comportamiento de la dinámica seguida por la población.

Sin embargo, también hay que notar que en los otros casos, mediante este método, durante las iteraciones, no se alcanzó convergencia alguna en los parámetros, debido por una parte, a que desde un principio se tuvo un valor óptimo para la función, en cuanto a la descripción de la estructura por edad de la PEA ocupada y las estructuras de la fecundidad por edad para los años 1952 y 1970; y por otra, porque al aplicar el ajuste correctivo mediante el método bilogístico en la descripción de la estructura de la fecundidad por edad para 1988, el patrón estándar empleado impuso su perfil, impidiendo alcanzar un óptimo para la función. Desde luego, sólo a través de probar el método iterativo y obtener la correlación entre los valores observados y los estimados por iteración es que se llegó a tales conclusiones.

Otro aspecto es que la función de Gompertz-Makeham permite la desagregación por edades o años individuales partiendo de la correspondiente agrupación quinquenal de edad e intervalos censales de 10 años; es decir, su aplicación después del ajuste descriptivo arrojó como resultado los estimados de población por cada año censal en un caso y en otro los componentes poblacionales de la PEA ocupada por edad.

Para la primera situación estudiada es sorprendente cómo después de lograr una magnifica descripción del crecimiento de la población en México para el periodo 1920-1990, al proyectar con la misma función de Gompertz-Makeham [óptima, además], los valores proyectados para los años 2000 y 2010 se dispararon completamente. Sin embargo, como ya se señaló, teniendo en 
mente el criterio demográfico, se aplicó una nueva función de Gompertz-Makeham que describiera tal fenómeno en el periodo 1960-1990; entonces la proyección resultó bastante congruente con las estimaciones esperadas de acuerdo con las nuevas realidades.

Lo importante en este caso es que se comprueba que una función continua, como la que aquí hemos utilizado para el periodo señalado (1960-1990), logra captar el impresionante cambio registrado en los ritmos de crecimiento de la población y permite proyectarlo con una muy aceptable precisión al menos, para el ya cercano año 2000. Esto nos sugiere que una vez que se cuente con las cifras finales del Censo de 1990, convendría efectuar nuevamente el ejercicio para afinar el valor estimado de la proyección.

Con respecto al estudio de las estructuras de la fecundidad por edad, comprobando previamente que la función de GompertzMakeham realiza un ajuste descriptivo adecuado, se pueden proyectar aceptablemente dichas estructuras.

Disponiendo de un nivel de fecundidad como meta, de acuerdo con los cambios demográficos actuales y después de observar las tendencias seguidas en el comportamiento de los parámetros de la función de Gompertz-Makeham en los años estudiados, se comprueba que al realizar la simulación matemática, variando el valor de dichos parámetros se obtienen posibles estructuras de la fecundidad por grupo de edad a manera de proyección.

En el caso de la estructura por edad de la PEA ocupada, las diversas alternativas producto de la simulación efectuada con los valores de los parámetros de la función de Gompertz-Makeham comprueban lo útil que resulta tal función matemática para visualizar diferentes escenarios en que podría darse la cambiante estructura por edad de la PEA ocupada.

Finalmente, como reflexión y conclusión, el trabajo muestra lo versátil, lo flexible y lo útil que resulta aplicar la función de Gompertz-Makeham, siempre y cuando se trabaje con el conocimiento y el empleo de las hipótesis y supuestos demográficos involucrados. "

\section{Bibliografía}

Bocaz, A. (1974), "El uso de la ley de Makeham como función demográfica", en Notas de Población, año II, vol. 6, Celade, pp. 37-64.

Cervera, M. y V. Partida (1977), Tablas de vida económicamente activa para la República Mexicana, Centro Nacional de Información y Estadísticas del Trabajo.

Chackiel, J. [1981), "El modelo de mortalidad de Brass", separata de No- 
tas de Población, año IX, núm. 25, Celade, pp. 93-94.

(1979), "Estructura de la fecundidad por edades: ajuste y proyección mediante la función de Gompertz línealizada", en Notas de Población, año viI, vol. 20, Celade.

Consejo Nacional de Población [1988], México Demográfico, Breviario, Conapo.

Dirección General de Estadística, Censos Generales de Población y Vivienda: IV, V, VI, VII, VIII, IX, X y XI, Dirección General de Estadística, 1921 a 1990.

Johnson R. y N. Johnson (1977), Survival models and data analysis, Nueva York, John Wiley and Sons.

Keyfitz, Nathan (1979), Introducción a las matemáticas de población, Santiago de Chile, Celade.

Leguina, Joaquín (1973), Fundamentos de demografía, México, Siglo XXI Editores.

Lotka, A. [1969], Teoría analítica de las asociaciones biológicas, Santiago de Chile, Celade.

Mina, A. y E. García [1984], "La función de Makeham en la descripción de la estructura por edad de la población económicamente activa", en Revista de Estadística y Geografía, vol. Iv, núm. 13, INEGI, pp. 49-69. (1989), "La función Gompertz-Makeham en el análisis actuarial y demográfico en México", en La Actuaría en México, Antología de algunos trabajos relevantes, Colegio Nacional de Actuarios.

Mood, Alexander M. (1965), Introducción a la teoría de la estadística, Madrid, Aguilar.

Newell, Colin (1988), Methods and Models in Demography, Nueva York, The Guilford Press.

ONU (1986), Técricas Indirectas de Estimación Demográfica, Manual X.

Secretaría de Programación y Presupuesto (1979), Encuesta Complementaria a la Encuesta Continua sobre Ocupación, México, diciembre. 